REPRESENTATION THEORY

An Electronic Journal of the American Mathematical Society

Volume 13, Pages 1-7 (January 21, 2009)

S 1088-4165(09)00344-6

\title{
ON THE CONNECTEDNESS OF DELIGNE-LUSZTIG VARIETIES
}

\author{
ULRICH GÖRTZ
}

\begin{abstract}
We give a criterion which determines when a union of one-dimensional Deligne-Lusztig varieties has a connected closure. We obtain a new, short proof of the connectedness criterion for Deligne-Lusztig varieties due to Lusztig.
\end{abstract}

\section{INTRODUCTION}

We fix a finite field $\mathbb{F}_{q}$ with $q$ elements and of characteristic $p$, and an algebraic closure $\mathbb{F}$ of $\mathbb{F}_{q}$. Let $G_{0}$ be a connected reductive group over $\mathbb{F}_{q}$, and let $T_{0} \subset B_{0} \subset$ $G_{0}$ be a maximal torus and a Borel subgroup, defined over $\mathbb{F}_{q}$. We denote by $G, B$, etc., the base change to $\mathbb{F}$, and we usually identify varieties over $\mathbb{F}$ with their sets of $\mathbb{F}$-valued points. Denote by $W$ the absolute Weyl group, and denote by $\sigma$ the Frobenius automorphism on $\mathbb{F}$ (and $G, W$, etc.). Let $S \subset W$ be the set of simple reflections determined by $B$. For each $w \in W$, we have the Deligne-Lusztig variety $X(w)$ over $\mathbb{F}$, defined by [2, Def. 1.4]:

$$
X(w):=\left\{g \in G / B ; g^{-1} \sigma(g) \in B w B\right\} .
$$

The unique zero-dimensional Deligne-Lusztig variety is $X(\mathrm{id})=\left(G_{0} / B_{0}\right)\left(\mathbb{F}_{q}\right)$, and $X(w)$ is one-dimensional if and only if $w \in S$. For $s \in S$, the closure $\overline{X(s)}$ of $X(s)$ is $X(s) \cup X$ (id). See Fact 2.1 below.

Theorem 1.1. Let $I \subseteq S$. The union

$$
\overline{X(I)}:=X(\mathrm{id}) \cup \bigcup_{s \in I} X(s)
$$

of Deligne-Lusztig varieties is connected if and only if I is not contained in any proper $\sigma$-stable subset of $S$.

Proof. If $I$ is contained in a proper $\sigma$-stable subset of $S$, then $\overline{X(I)}$ projects onto the set of rational points in the quotient of $G$ by the corresponding parabolic subgroup, so it cannot be connected. The converse will be proved in Section 3 .

If $G_{0}$ is a unitary group, the theorem was proved by Ekedahl and van der Geer, 44. Lemma $7.6 \mathrm{ii})$ ], and applied to study the moduli space of principally polarized abelian varieties over $\mathbb{F}_{q}$. See also [7, Lemma 7.1] and the discussion following it, and the remarks below. For $G_{0}=G L_{n}$, the theorem amounts to the statement that one can pass between any two $\mathbb{F}_{q}$-rational flags by a series of steps where in

Received by the editors September 19, 2008 and, in revised form, December 8, 2008.

2000 Mathematics Subject Classification. Primary 14L35; Secondary 20 G40.

The author was partially supported by a Heisenberg grant and by the SFB/TR 45 "Periods, Moduli Spaces and Arithmetic of Algebraic Varieties" of the DFG (German Research Foundation).

(C)2009 American Mathematical Society Reverts to public domain 28 years from publication 
each step only one subspace of the flag is modified. It is not hard to see that this is true (this case is also contained in [4]).

Corollary 1.2. For $w \in W$ the following are equivalent:

(i) $X(w)$ is irreducible.

(ii) The closure $\overline{X(w)}$ is connected.

(iii) The element $w$ is not contained in any $\sigma$-stable proper standard parabolic subgroup of $W$.

This result was first obtained by Lusztig ([12, Example $3.10(\mathrm{~d})]$ ); now there are several proofs in the literature by Digne and Michel [3. Prop. 8.4], Bonnafé and Rouquier [1, Thm. 2], and Lusztig [13, Lemma 7.14]. The proof given below is self-contained. For instance, in contrast to [1, we do not use the fact that Deligne-Lusztig varieties for a Coxeter element are connected (which was proved by Deligne and Lusztig, see [11) as an ingredient. Note that it is easy to generalize the criterion to Deligne-Lusztig varieties in quotients $G / P$ by parabolic subgroups; see [1].

Proof. Since the closure $\overline{X(w)}$ is normal (Fact 2.1 (2)), (i) and (ii) are equivalent. As in the theorem, it is easy to see that (ii) implies (iii). Finally, assume that (iii) holds, and let $I(w)=\{s \in S ; s \leq w\}$. The hypothesis says that $I(w)$ is not contained in a $\sigma$-stable proper subset of $S$, so by the theorem $\overline{X(I(w))}$ is connected. By Fact 2.1 (3), every connected component of $\overline{X(w)}$ contains a rational point, so every connected component meets $\overline{X(I(w))}$, and (ii) follows.

A special case of the theorem is applied by $\mathrm{Yu}$ and the author [7] to study the moduli space (over $\mathbb{F}_{p}$ ) of $g$-dimensional abelian varieties with Iwahori level structure at $p$. On this space, one has the Kottwitz-Rapoport stratification, given by the relative position of the chain of first de Rham cohomology groups and the chain of Hodge filtrations. This relative position is an element in the extended affine Weyl group of $G S p_{2 g}$. It is striking that Kottwitz-Rapoport strata share many properties with Deligne-Lusztig varieties: they are quasi-affine, their closures are normal, and the closure relations are given by the Bruhat order. Moreover, the proofs are, in a sense, quite similar. For the quasi-affineness in the case of KR strata, one uses that the moduli space with Iwahori level structure maps under a finite morphism to a flag bundle over the moduli space of principally polarized abelian varieties. On the latter space, the Hodge bundle is ample. The KR strata map to "relative Deligne-Lusztig varieties" in this flag bundle. One shows in a similar way as in Haastert's proof of the quasi-affineness of Deligne-Lusztig varieties (see [8]) that the strata are quasi-affine; see [7, Thm. 5.4]. To determine the closure relations, one can use a "local model diagram": in the case of Deligne-Lusztig varieties, the local structure is the same as the local structure of Schubert cells (and for their closures etc., see the proof of Fact 2.1 (1), (2) below). For Kottwitz-Rapoport strata, the local structure is described by the local model (see Rapoport and Zink's book [16, Ch. 3]) which can be identified with a union of Schubert varieties in an affine flag variety [5]. See also the survey articles by Rapoport [15] and Haines [9].

Those Kottwitz-Rapoport strata which are contained in the supersingular locus are actually disjoint unions of Deligne-Lusztig varieties (by [6, Cor. 6.5] and [7. Cor. 7.4]). Using the theorem above, one can show that all KR strata which are not contained in the supersingular locus, are connected (and conversely, if the level 
structure away from $p$ is small enough), 7, Thm. 7.3]. See also [4 for a similar application. The reasoning in the proof of the corollary also appears in Oort's paper 14] about the canonical stratification (now called the Ekedahl-Oort stratification) of the moduli space of principally polarized abelian varieties.

One can hope that this approach also yields results about the connectedness of closed affine Deligne-Lusztig varieties in the Iwahori case (see Viehmann 18] for the case of a maximal parahoric subgroup). However, an additional effort is required because very little is known about properties of affine Deligne-Lusztig varieties, for instance, whether each connected component contains a rational point in its closure.

Acknowledgments. I am grateful to Tetsushi Ito for his comments on the text, and to George Lusztig and Michael Rapoport for pointing me to the references [13] and [12], resp. Furthermore, I thank the referee for her/his remarks.

\section{Basic properties of Deligne-Lusztig varieties}

We regard the set $\left(G_{0} / B_{0}\right)\left(\mathbb{F}_{q}\right)$ as a subset of $G / B$; it is the set of points fixed by $\sigma$, and as usual we call its elements the rational points of $G / B$.

On $W$, we have the Bruhat order $\leq$, and the length function $\ell$. We denote by $C_{v}:=B v B / B$ the Schubert cell associated with $v \in W$. Its closure $\bar{C}_{v}$ is called the Schubert variety for $v$. The set $C_{v} \cap\left(G_{0} / B_{0}\right)\left(\mathbb{F}_{q}\right)$ is non-empty if and only if $v$ is fixed by $\sigma$. In this case $C_{v}$ is defined over $\mathbb{F}_{q}$, and is isomorphic to $\mathbb{A}^{\ell(v)}$ over $\mathbb{F}_{q}$, and we denote its set of rational points by $C_{v}\left(\mathbb{F}_{q}\right)$. We denote the subgroup of $W$ of elements fixed by $\sigma$ by $W^{\sigma}$.

We need the following well-known properties of Deligne-Lusztig varieties:

Fact 2.1. (1) Let $w \in W$. We have $\overline{X(w)}=\bigcup_{v \leq w} X(v)$.

(2) Let $v \leq w$ be elements of $W$. Fix points $v_{1} \in X(v)(\mathbb{F}), v_{2} \in C_{v}(\mathbb{F})$. Then the singularities of $\overline{X(w)}$ in $v_{1}$ and of $\bar{C}_{w}$ in $v_{2}$ are smoothly equivalent. In particular, $\overline{X(w)}$ is normal.

(3) Let $w \in W$, and let $X \subseteq X(w)$ be an irreducible component. Then the closure $\bar{X}$ contains a rational point of $G / B$.

Proof. To make this paper self-contained, we sketch proofs of these facts. For (1), (2), we repeat the proof given in [6, Section 5]. Let $L: G \rightarrow G$ be the Lang map $g \mapsto g^{-1} \sigma(g)$. The composition $G \stackrel{L}{\longrightarrow} G \stackrel{\mathrm{pr}}{\longrightarrow} G / B$ is $B$-equivariant if $B$ acts in $G$ by right multiplication, and on $G / B$ by $b . g B=b^{-1} g B$. Taking the quotient, we get a smooth morphism of relative dimension $\operatorname{dim} B$,

$$
G / B \rightarrow[B \backslash G / B],
$$

from $G / B$ to the stack quotient $[B \backslash G / B]$. The underlying topological space of this stack is just the Weyl group $W$, and the fiber over $w \in W$ is $X(w)$. This proves the first two assertions.

To prove (3), it is enough to show (because of (1)) that $G_{0}\left(\mathbb{F}_{q}\right)$ acts transitively on the set of connected components of $X(w)$. We recall the argument presented in [1]: the Lang map $L$ is a $G_{0}\left(\mathbb{F}_{q}\right)$-torsor, so $G_{0}\left(\mathbb{F}_{q}\right) \backslash L^{-1}(B w B) \cong B w B$ is connected. On the other hand, $G_{0}\left(\mathbb{F}_{q}\right) \backslash L^{-1}(B w B)$ surjects onto $G_{0}\left(\mathbb{F}_{q}\right) \backslash X(w)$. 
Remark 2.2. Another way to prove Fact (3) is to use that all Deligne-Lusztig varieties are quasi-affine, as was proved by Haastert [8]. In fact, a quasi-affine subvariety of $G / B$ can only be closed if it is finite. One concludes using (1) and induction on the dimension. This makes the analogy with [7] even closer: In loc. cit., this argument is used to prove that every irreducible component of a KR stratum has a point of the minimal (0-dimensional) stratum in its closure, after first proving that all KR strata are quasi-affine. The proof of quasi-affineness relies on the same principle as Haastert's proof. Similarly, as we will do for Deligne-Lusztig varieties below, one can then exploit the fact that every closure of an irreducible component of a stratum meets the minimal stratum, in order to give a connectedness criterion for KR strata. At this point, as well as for the proof of quasi-affineness, one combines results about the moduli space $\mathcal{A}_{g}$ of principally polarized abelian varieties with results about flag varieties or, more specifically, Deligne-Lusztig varieties. The latter come in in order to deal with the fibers of the projection from the moduli space with Iwahori level structure to $\mathcal{A}_{g}$.

\section{Proof of the CONNECTEDNESS CRITERION}

Lemma 3.1. Let $s \in S$.

(1) Let $v \in W$. Then $X(s) \cap C_{v}$ is empty, unless $v \in W^{\sigma}$ and $v s<v$, in which case the intersection is equi-dimensional of dimension 1.

(2) Let $v \in W^{\sigma}$, such that $v s<v$, and let $x \in C_{v}\left(\mathbb{F}_{q}\right)$. Then there exists an irreducible component $X \subset X(s)$ which is contained in $C_{v}$ and whose closure $\bar{X}$ (which is an irreducible component of $\overline{X(s)}$ ) contains $x$. Furthermore, $\bar{X}$ contains a rational point of some cell $C_{v^{\prime}}, v^{\prime} \in W^{\sigma}, v^{\prime}<v$.

Proof. Denote by $U$ the unipotent radical of $B$, and by $U^{-}$the unipotent radical of the Borel opposite to $B$. We let $U_{v}=v U^{-} v^{-1} \cap U$, and then have an isomorphism $U_{v} \rightarrow C_{v}, g \mapsto g v$. It induces an isomorphism

$$
X(s) \cap C_{v} \stackrel{\cong}{\longrightarrow}\left\{g \in U_{v} ;(g v)^{-1} \sigma(g v) \in B s B\right\}=: U_{v}(s) .
$$

Assume that this set is non-empty. Since $U$ is defined over $\mathbb{F}_{q}$, we have the Lang map $L: U \rightarrow U, u \mapsto u^{-1} \sigma(u)$, and $L$ maps $U_{v}(s)$ into $v B s B \sigma\left(v^{-1}\right) \cap U$. If this set is non-empty, then $\sigma(v) \in B v B s B$, which shows that $\sigma(v)=v$, and that $v s<v$.

This means in particular that $U_{v}$ is defined over $\mathbb{F}_{q}$, so that the Lang map $L$ restricts to a (surjective, finite, étale) morphism $U_{v} \rightarrow U_{v}$, and further restricts to a surjection

$$
U_{v}(s) \stackrel{L}{\longrightarrow} v B s B v^{-1} \cap U_{v} \stackrel{\cong}{\longrightarrow} B s B \cap v^{-1} U v \cap U^{-},
$$

where the second morphism is conjugation by $v^{-1}$. The Bruhat decomposition shows that the target of this morphism is irreducible of dimension 1 in the latter case. Because $L$ is finite étale, (1) is proved.

Now consider $v, x$ as in (2). Let $X^{\prime} \subset X(s)$ be an irreducible component which meets $C_{v}$. It follows from part (1) that $X^{\prime} \cap C_{v}$ has dimension 1. We claim that $X^{\prime} \subseteq C_{v}$. Otherwise, the zero-dimensional complement $X^{\prime} \backslash C_{v}$ would intersect some other Schubert cell $C_{v^{\prime}}$, but this is impossible by (1), applied to $v^{\prime}$. Furthermore, denoting by $\overline{X^{\prime}}$ the closure of $X^{\prime}$, we have, similarly as above, a finite (étale) map $\overline{X^{\prime}} \cap C_{v} \subset \overline{X(s)} \cap C_{v} \longrightarrow(B s B \cup B) \cap v^{-1} U v \cap U^{-}=\left(B s B \cap v^{-1} U v \cap U^{-}\right) \cup\{1\}$. 
Its image is one-dimensional and closed, and hence equal to the right-hand side. In particular, $\overline{X^{\prime}} \cap C_{v}\left(\mathbb{F}_{q}\right)$, which is the fiber over 1 , is non-empty. By applying a suitable element of $B\left(\mathbb{F}_{q}\right)$ to $X^{\prime}$ we produce an irreducible component $X \subset X(s)$ which is contained in $C_{v}$ and whose closure contains $x$.

Because $\bar{X}$ is projective, it is not contained in $C_{v}$, so it intersects non-trivially with some cell $C_{v^{\prime}}$ in the closure of $C_{v}$; since $X \subset C_{v}$, all points in $\bar{X} \backslash C_{v}$ lie in $X($ id), i.e. are rational points of $G / B$.

We note that part (1) of the lemma, except for the equi-dimensionality statement, follows from (a very special case of) Prop. 8.2 in [3], and the proof given above is basically the relevant part of the proof in loc. cit.

Proposition 3.2. Let $I \subseteq S$ be a subset which is not contained in a $\sigma$-stable proper subset of $S$. Let $x \in\left(G_{0} / B_{0}\right)\left(\mathbb{F}_{q}\right)$ be a rational point, say $x \in C_{v}$. Assume $v \neq \mathrm{id}$. Then there exists $s \in I$ such that $v s<v$. For each such $s$, there exist $v^{\prime}<v$ and a rational point in $C_{v^{\prime}}\left(\mathbb{F}_{q}\right)$ which lies in the same connected component of $\overline{X(I)}$ as $x$.

Proof. To show the existence of $s$, note that $C_{v} \cap\left(G_{0} / B_{0}\right)\left(\mathbb{F}_{q}\right) \neq \emptyset$ implies that $v \in W^{\sigma}$, so in particular, $v s<v$ if and only if $v \sigma(s)<v$. This implies that unless $v=\mathrm{id}$, there exists $s \in I$ as desired. Now we apply part (2) of the lemma.

By induction, the proposition implies that every rational point of $\left(G_{0} / B_{0}\right)\left(\mathbb{F}_{q}\right)$ is in the same connected component of $\overline{X(I)}$ as the point $C_{\text {id. }}$. Because every irreducible component of $\overline{X(I)}$ contains a rational point (Fact $2.1(3)$ ), the theorem is proved.

\section{More Detailed analysis}

Let $s \in S$, and let $X_{1} \subseteq X(s)$ be the irreducible component whose closure contains $C_{\text {id }}$; it is unique because the closure of $X(s)$ is normal, so its irreducible components do not intersect. If the group $G$ is split, the situation is relatively simple: $\overline{X_{1}}=\bar{C}_{s}$. In particular, all irreducible components of $\overline{X(s)}$ are just projective lines, and contain $q+1$ rational points. The finite group $G_{0}\left(\mathbb{F}_{q}\right)$ acts on $\overline{X(s)}$, and given $v$ with $v s<v, v s X_{1}$ is an irreducible component of $\overline{X(s)}$ which connects a rational point in $C_{v}$ with one in $C_{v s}$, as in the proposition. We also see that $X(s)$ has $\#\left(G_{0} / B_{0}\right)\left(\mathbb{F}_{q}\right) /(1+q)$ irreducible components. In general, the picture is to some extent similar:

For $s \in S$, we denote by $W^{s}$ the subgroup generated by all elements $\sigma^{i}(s), i \in \mathbb{Z}$, and by $w_{0}^{s}$ its longest element. In particular, $w_{0}^{s}=s$ if $G$ is split. In every case, $w_{0}^{s} \in W^{\sigma}$. We need to know the structure of $W^{\sigma}$, as described in

Lemma 4.1 (Steinberg [17, §1]; Kottwitz, Rapoport [10, Prop. 2.3]). The subgroup $W^{\sigma} \subseteq W$ together with the set $\left\{w_{0}^{s} ; s \in S\right\}$ is a Coxeter system. The Bruhat order on $W^{\sigma}$ is the restriction of the Bruhat order on $W$ to $W^{\sigma}$.

In particular, the lemma implies that $W^{s} \cap W^{\sigma}=\left\{\mathrm{id}, w_{0}^{s}\right\}$, and that for $v \in W^{\sigma}$, $v s<v$ if and only if $v w_{0}^{s}<v$. Using this and reduction to a Levi subgroup, we can use Proposition 3.2 to describe the irreducible components of one-dimensional Deligne-Lusztig varieties in the general case, as follows: 
Proposition 4.2. Let $s \in S$.

(1) Let $X_{1} \subset X(s)$ be the irreducible component whose closure contains the point $C_{\mathrm{id}}$. Then $\overline{X_{1}} \cap\left(G_{0} / B_{0}\right)\left(\mathbb{F}_{q}\right)=C_{\mathrm{id}} \cup C_{w_{0}^{s}}\left(\mathbb{F}_{q}\right)$.

(2) Let $v \in W^{\sigma}$ such that $v s<v$. Then $v w_{0}^{s}<v$. For every $x \in C_{v}\left(\mathbb{F}_{q}\right)$, there exists an irreducible component of $X(s)$ whose closure contains $x$ and a rational point of $C_{v w_{0}^{s}}$.

Furthermore, we get a formula for the number of connected components. We extend the notation introduced above as follows: For $w \in W$, the subgroup $W^{w}$ denotes the smallest $\sigma$-stable standard parabolic subgroup of $W$ which contains $w$, i.e. the subgroup generated by all $W^{s}, s \leq w$. We denote by $P_{0}^{w}$ the standard parabolic subgroup of $G_{0}$ corresponding to $W^{w}$. For a subset $H \subseteq W$, let $N(H)=\sum_{w \in H \cap W^{\sigma}} q^{\ell(w)}$. For instance, $N(W)=\#\left(G_{0} / B_{0}\right)\left(\mathbb{F}_{q}\right)$, and more generally $N\left(W^{w}\right)=\#\left(P_{0}^{w} / B_{0}\right)\left(\mathbb{F}_{q}\right)$. Concretely, for $s \in S$ we have $N\left(W^{s}\right)=1+q^{\ell\left(w_{0}^{s}\right)}$.

Proposition 4.3. Let $w \in W$. The projection $G / B \rightarrow G / P^{w}$ restricts to a surjection $X(w) \rightarrow\left(G_{0} / P_{0}^{w}\right)\left(\mathbb{F}_{q}\right)$, whose fibers are the connected components of $X(w)$. In particular, $X(w)$ has $N(W) / N\left(W^{w}\right)$ connected components, and every connected component has $N\left(W^{w}\right)$ rational points of $G / B$ in its closure.

Proof. This is an easy consequence of the above. See [1] or [6, Cor. 5.3].

\section{REFERENCES}

[1] C. Bonnafé and R. Rouquier, On the irreducibility of Deligne-Lusztig varieties, C. R. A. S. 343 (2006), 37-39. MR2241956 (2007a:14055)

[2] P. Deligne and G. Lusztig, Representations of reductive groups over finite fields, Annals of Math. 103 (1976), 103-161. MR.0393266 (52:14076)

[3] F. Digne and J. Michel, Endomorphisms of Deligne-Lusztig varieties, Nagoya Math. J. 183 (2006), 35-103. MR2253886(2007e:20026)

[4] T. Ekedahl, G. van der Geer, Cycle classes of the E-O stratification on the moduli of abelian varieties, arXiv:math.AG/0412272v2.

[5] U. Görtz, On the flatness of local models for the symplectic group, Adv. Math. 176 (2003), 89-115. MR1978342 (2004d:14023)

[6] U. Görtz, C.-F. Yu, Supersingular Kottwitz-Rapoport strata and Deligne-Lusztig varieties, arXiv:math/0802.3260v2.

[7] U. Görtz, C.-F. Yu, The supersingular locus in Siegel modular varieties with Iwahori level structure, arXiv:0807.1229v2.

[8] B. Haastert, Die Quasiaffinität der Deligne-Lusztig-Varietäten, J. Alg. 102 (1986), 186-193. MR853238 (87i:20086)

[9] T. Haines, Introduction to Shimura varieties with bad reduction of parahoric type, in: Harmonic analysis, the trace formula, and Shimura varieties, 583-642, Clay Math. Proc., 4, Amer. Math. Soc., 2005. MR2192017 (2006m:11085)

[10] R. E. Kottwitz and M. Rapoport, Minuscule alcoves for $\mathrm{GL}_{n}$ and $\mathrm{GSp}_{2 n}$, Manu. Math. 102 (2000), 403-428. MR.1785323 (2001g:20059)

[11] G. Lusztig, Coxeter orbits and eigenspaces of Frobenius, Invent. Math. 38 (1976), 101-159. MR 0453885 (56:12138)

[12] G. Lusztig, Representations of finite Chevalley groups, Expository Lectures from the CBMS Regional Conference held at Madison, Wis., 1977. CBMS Regional Conf. Series in Math., 39. Amer. Math. Soc., 1978. MR518617 (80f:20045)

[13] G. Lusztig, A class of perverse sheaves on a partial flag manifold, Represent. Theory 11 (2007), 122-171. MR2336607 (2008j:20151)

[14] F. Oort, A stratification of a moduli space of abelian varieties, in: Moduli of abelian varieties (Texel Island, 1999), Progr. Math. 195, 255-298, Birkhäuser 2001. MR.1827027(2002b:14055)

[15] M. Rapoport, A guide to the reduction modulo $p$ of Shimura varieties, in: Automorphic forms. I. Astérisque 298 (2005), 271-318. MR2141705 (2006c:11071) 
[16] M. Rapoport and Th. Zink, Period Spaces for p-divisible groups. Annals of Math. Studies 141, 1996. MR1393439 (97f:14023)

[17] R. Steinberg, Endomorphisms of linear algebraic groups, Mem. Amer. Math. Soc. 80 (1968), 1-108. MR.0230728 (37:6288)

[18] E. Viehmann, Connected components of closed affine Deligne-Lusztig varieties, Math. Ann. 340 (2008), 315-333. MR2368982

Mathematisches Institut, Beringstr. 1, 53115 Bonn, Germany

E-mail address: ugoertz@math.uni-bonn.de 